\title{
Determination of bounds on failure probability in the presence of hybrid uncertainties
}

\author{
M B ANOOP* and K BALAJI RAO \\ Structural Engineering Research Centre, CSIR Campus, Taramani, \\ Chennai 600113 \\ e-mail: balajiserc1@yahoo.com
}

MS received 30 January 2008; revised 11 July 2008

\begin{abstract}
A fundamental component of safety assessment is the appropriate representation and incorporation of uncertainty. A procedure for handling hybrid uncertainties in stochastic mechanics problems is presented. The procedure can be used for determining the bounds on failure probability for cases where failure probability is a monotonic function of the fuzzy variables. The procedure is illustrated through an example problem of safety assessment of a nuclear power plant piping component against stress corrosion cracking, considering the stochastic evolution of stress corrosion cracks with time. It is found that the bounds obtained enclose the values of failure probability obtained from probabilistic analyses.
\end{abstract}

Keywords. Safety assessment; probability of failure; fuzzy sets; possibility measure; necessity measure.

\section{Introduction}

A fundamental component of safety assessment of complex engineering facilities, such as nuclear power plants, is the appropriate representation and incorporation of uncertainty in different variables (Helton \& Oberkampf 2004). Uncertainty can be classified into two main types, namely, aleatory (random or irreducible or Type A) uncertainty and epistemic (reducible or Type B) uncertainty (Nikolaidis \& Haftka 2001). Aleatory uncertainty arises due to inherent randomness in physical phenomenon or processes, and epistemic uncertainty arises due to lack of knowledge about the quantities. While probability theory has been traditionally used to represent both types of uncertainties, various researchers have pointed out that it may not be proper to use probability theory to represent epistemic uncertainty in the presence of limited knowledge (Helton \& Oberkampf 2004, Chen et al 1999). A number of alternative theories, such as fuzzy set theory, evidence theory, convex modelling, and interval analysis, for modelling epistemic uncertainties have been proposed by various researchers (Helton \& Oberkampf 2004). In particular, fuzzy set theory provides a more rational framework for handling uncertainties arising from vagueness, namely, imprecision of definition or use of

${ }^{*}$ For correspondence 
linguistic terms in a natural or artificial language (Ross 1995). The benefits of fuzzifying uncertain variables include greater generality, higher expressive power, an enhanced ability to model real world problems, and a methodology for exploiting tolerance for imprecision (Ross 1995).

Savoia (2002) proposed a procedure for structural reliability analysis using the framework of possibility theory, and has shown that the proposed procedure gives conservative values for small or large fractiles of output variables when compared to that obtained using probability theory. But Savoia (2002) considered only the fuzzy uncertainties, while in real world problem both probabilistic and fuzzy uncertainties co-exist. Thus, there is a need to develop special techniques, which can handle hybrid uncertainties (i.e. fuzzy and random), for carrying out safety assessment. Different methods have been proposed by various researchers for handling fuzzy and random uncertainties together (Haldar \& Reddy 1992, Dubois et al 1993, Anoop et al 2006, Anoop et al 2008). One method is to determine equivalent probability distributions for the fuzzy sets (or vice versa), and carry out the analysis in the framework of probability theory (or fuzzy set theory) (Dubois et al 1993, Anoop et al 2006). But, according to possibility theory, if the membership function of the fuzzy set is the only available information, there is a class of probability measures which are equally valid (Savoia 2002). Hence, it is not adequate to represent the fuzzy set using a single equivalent probability distribution in the reliability analysis. In the present study, a procedure to determine the bounds on failure probability compatible with the available data, considering both probabilistic and fuzzy uncertainties, is proposed. The procedure is based on possibility theory, and can be used for calculating the bounds on failure probability for stochastic systems.

The paper is organized as follows. A brief introduction to possibility theory is given in the next section. The proposed procedure for determination of bounds on failure probability in the presence of probabilistic and fuzzy uncertainties is presented in $\S 3$. The procedure is illustrated through an example problem of safety assessment of a nuclear power plant piping component subjected to stress corrosion cracking in $\S 4$. The results and discussions are given in $\S 5$, and the conclusions are given in $\S 6$.

\section{Possibility theory}

Theory of possibility is based on two measures of confidence, namely, possibility measure ( $\Pi$ ) and necessity measure $(N)$. A confidence measure, $g(A)$, is a number $0 \leq g(A) \leq 1$, which represents the confidence one has on the occurrence of event $A \subseteq \Omega$, where $\Omega$ is the sure event (Savoia 2002). While several kinds of confidence measures can be defined, depending on the amount and the type of information available, all confidence measures must satisfy at least the axiom of monotonicity with respect to set inclusion. That is,

$$
A \subseteq B \Rightarrow g(A) \leq g(B)
$$

If the membership function of a fuzzy set $X$, defined over $\Omega$, is the available information, the possibility and necessity measures, associated with the occurrence of an event $A \subseteq \Omega$, are defined as (Savoia 2002, Ferrari \& Savoia 1998, Dubois et al 1999):

$$
\begin{aligned}
& \Pi(A)=\sup _{\omega \in A} \pi_{x}(\omega) \\
& N(A)=\inf _{\omega \in \bar{A}}\left(1-\pi_{x}(\omega)\right)=1-\Pi(\bar{A}),
\end{aligned}
$$


where $\pi_{x}$ is the possibility distribution of $X$. Since it is assumed that membership function of the fuzzy set $X$ is available, the same can be considered as the possibility distribution of $X$. In equation (3), $\bar{A}$ denotes complement of event $A$. It has been shown by Dubois \& Prade (1980) and Savoia (2002) that possibility and necessity are limit cases of an equivalence class of probability distributions compatible with available data. This is in line with the consistency principle proposed by Zadeh which has been translated by Dubois \& Prade (1980) as: 'the degree of possibility of an event is greater than or equal to its degree of probability, which must be itself greater than or equal to its degree of necessity'. Thus, an equivalence class $\mathcal{P}$ of probability measures $P$ compatible with available data can be defined as:

$$
\{P \mid \forall A, N(A) \leq P(A) \leq \Pi(A)\} .
$$

Without any additional information, all probability measures defined by equation (4) are equally valid (Savoia 2002, Ferrari \& Savoia 1998).

Considering a fuzzy variable $Q$ with membership function $\mu_{Q}(x)$ and defining an event $A$ as $A=(-\infty, x]$, the following cumulative density functions (CDF) can be defined:

$$
\begin{aligned}
& F_{*}(x)=N((-\infty, x]) \\
& F^{*}(x)=\Pi((-\infty, x]) .
\end{aligned}
$$

From equation (4), it is noted that the CDFs defined by equation (5) represent lower- and upper-bound for all probability measures $P$ belonging to class $\mathcal{P}$, compatible with available data. Thus,

$$
F_{*}(x) \leq F(x) \leq F^{*}(x) ; F(x)=P((-\infty, x]) .
$$

Using equations (2) and (3), $F_{*}(x)$ and $F^{*}(x)$ can be rewritten in terms of membership function of fuzzy set $Q$ as:

$$
\begin{aligned}
& F_{*}(x)=\inf \left\{1-\mu_{Q}(\omega) / \omega>x\right\} \\
& F^{*}(x)=\sup \left\{\mu_{Q}(\omega) / \omega \leq x\right\} .
\end{aligned}
$$

Thus, from the membership function of the fuzzy variable, one can determine the CDFs corresponding to the lower-bound $\left(F_{*}(x)\right)$ and upper-bound $\left(F^{*}(x)\right)$ of $X$, representing the bounds for all probability measures compatible with available information on $X$ (figure 1). Each of these CDFs can be used in reliability analysis to compute the respective values of probability of failure $\left(P_{F}\right)$ for a specified value of $X$. For cases where $P_{F}$ is a monotonic function of variable $X$, the values of failure probability obtained using the CDFs $F_{*}(x)$ and $F^{*}(x)$ will give the bounds on $P_{F}$. The proposed procedure for determining the bounds on $P_{F}$ in the presence of probabilistic and fuzzy uncertainties is given in the next section.

\section{Proposed procedure}

(i) Identify the random variables $\left(X_{r}^{1}, X_{r}^{2}, \ldots, X_{r}^{n}\right)$ and fuzzy variables $\left(X_{f}^{1}, X_{f}^{2}, \ldots, X_{f}^{m}\right)$ based on source and type of uncertainties.

(ii) For each random variable, $X_{r}^{i}$, identify the type of probability distribution and the values of the associated parameters. 


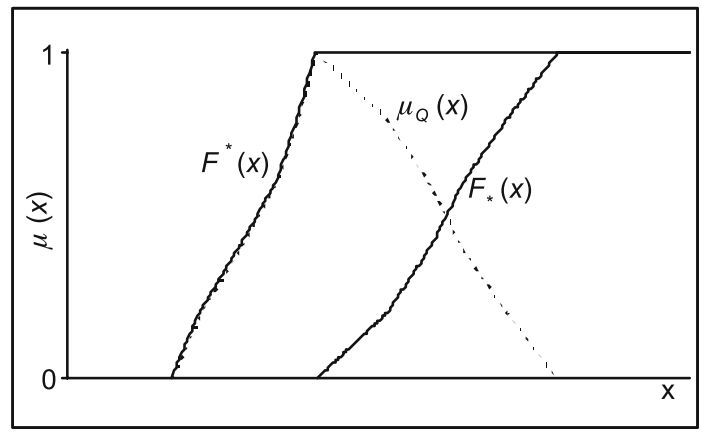

Figure 1. Bounds for probability measures compatible with the available information.

(iii) For each fuzzy variable, $X_{f}^{i}$, identify the suitable membership function, $\mu_{x_{f}^{i}}$. For engineering applications, to reduce computational complexity, fuzzy sets with triangular or trapezoidal form are most commonly used (Anoop et al 2006).

(iv) For each fuzzy variable, $X_{f}^{i}$, determine the lower-bound $\mathrm{CDF}, F_{*}\left(x_{f}^{i}\right)$, and the upperbound $\mathrm{CDF}, F^{*}\left(x_{f}^{i}\right)$, using equation (7).

(v) Carry out the reliability analysis considering each combination of $F_{*}\left(x_{f}^{i}\right)$ and $F^{*}\left(x_{f}^{i}\right)$ for the $m$ fuzzy variables along with the random variables $\left(X_{r}^{1}, X_{r}^{2}, \ldots, X_{r}^{n}\right)$ together (total number of combinations to be considered $\left.{ }^{1}=2^{m}\right)$, and compute the respective values of $P_{F}$.

(vi) Determine the bounds for failure probability as the minimum $\left(P_{F, \min }\right)$ and maximum $\left(P_{F, \max }\right)$ values from the $2^{m}$ values of $P_{F}$ obtained.

Computation of values of $P_{F}$ in Step 5 involves repeated application of reliability analysis techniques such as FORM, SORM. It is known that application of FORM/SORM for time variant reliability analysis problems with resistance degradation would involve restrictive assumptions regarding resistance and loading process types so that the problem can be solved within analytical framework. However, such difficulties can be overcome by Monte Carlo simulation techniques, and hence in this study, Monte Carlo simulation technique is used for computing the values of $P_{F}$. It is realised that Monte Carlo simulation technique is computationally intensive. An example problem of safety assessment of a nuclear power plant piping component against stress corrosion cracking, considering stochastic evolution of stress corrosion cracks with time, is provided in the next section to illustrate the proposed procedure.

\section{Example}

Stress corrosion cracking (SCC) is an important degradation mechanism to be considered for safety assessment of nuclear piping components made of austenitic steels. Safety assessment of a typical piping component made of AISI 304 stainless steel against SCC is carried out using proposed procedure. The sample problem, given as SCC baseline case in PRAISE manual (Harris et al 1992), is considered for this purpose. The piping component has an inner radius

\footnotetext{
${ }^{1}$ For each fuzzy variable, two combinations need to be considered; one corresponding to the upper-bound CDF and the other corresponding to the lower-bound CDF. Hence the total number of combinations to be considered is $2 \times 2 \times \cdots \times 2(m$ times $)=2^{m}$.
} 
Table 1. Pipe geometry, material properties and environmental conditions.

\begin{tabular}{ll}
\hline Pipe wall thickness $(\mathrm{m})$ & $0 \cdot 021$ \\
Inner diameter $(\mathrm{m})$ & $0 \cdot 364$ \\
Operating temperature $\left({ }^{\circ} \mathrm{C}\right)$ & $288 \cdot 0$ \\
Oxygen concentration $(\mathrm{ppm})$ & $0 \cdot 20$ \\
Water conductivity $(\mu \mathrm{s} / \mathrm{cm})$ & $0 \cdot 20$ \\
Pipe material & AISI 304 stainless steel \\
Modulus of elasticity, $E(\mathrm{MPa})$ & $1.83 \times 10^{5}$ \\
\hline
\end{tabular}

of $181.86 \mathrm{~mm}$ and a thickness of $21.34 \mathrm{~mm}$. Stress due to dead weight and thermal expansion is $70.38 \mathrm{MPa}$ and the operating pressure is $8.63 \mathrm{MPa}$. Total tensile stress in the pipe due to dead weight, thermal expansion and operating pressure is $105.09 \mathrm{MPa}$. It is assumed that there are no residual stresses. The approximate water temperature $(T)$ and average oxygen concentration $\left(\mathrm{O}_{2}\right)$ at steady state condition are $288^{\circ} \mathrm{C}$ and $0.20 \mathrm{ppm}$, respectively. The inputs related to geometry and material properties of the pipe and operating conditions are given in table 1 .

\subsection{Modelling stress corrosion cracking}

The methodology recommended in PRAISE (Harris et al 1992) for modelling SCC in pipe is followed in this study. In PRAISE, occurrence of SCC is modelled by considering it as a two-stage process, namely, (1) crack initiation and (2) crack propagation. Methodology recommended in PRAISE for modelling SCC is briefly described below.

4.1a Time to initiation: Time to initiation of stress corrosion crack is considered as a function of damage parameter, $D$, which represents effects of loading, environment and material variables on SCC. The damage parameter is given by

$$
D=f_{1} \text { (material) } \cdot f_{2} \text { (environment) } \cdot f_{3} \text { (loading), }
$$

where $f_{1}, f_{2}$ and $f_{3}$ are given by

$$
f_{1}=C_{1}(P a)^{C_{2}},
$$

where $P a$ is a measure of degree of sensitization, given by EPR (Electrochemical Potentiokinetic Reactivation) (in $\mathrm{C} / \mathrm{cm}^{2}$ ).

$$
f_{2}=O_{2}^{C_{3}} \exp \left[C_{4} /(T+273)\right] \log \left(C_{5} \gamma^{C_{6}}\right)
$$

where $O_{2}$ is oxygen concentration in ppm, $T$ is temperature in degrees centigrade and $\gamma$ is water conductivity in $\mu \mathrm{s} / \mathrm{cm}$.

The loading term $f_{3}$ is considered to be a function of stress. For constant applied load case, $f_{3}$ is given by

$$
f_{3}=\left(C_{8} \sigma^{C_{9}}\right)^{C_{7}}
$$

where $\sigma$ is stress in ksi.

$C_{1}$ to $C_{9}$ are constants whose values depend on type of material, and are evaluated by applying curve-fitting procedures to laboratory and field data. For AISI 304 austenitic stainless 
steel, values of these constants are given by $C_{1}=23 \cdot 0, C_{2}=0 \cdot 51, C_{3}=0 \cdot 18, C_{4}=$ $-1123 \cdot 0, C_{5}=8.7096, C_{6}=0.35, C_{7}=0.55, C_{8}=2.21 \times 10^{-15}$ and $C_{9}=6.0$ (Harris et al 1992).

In order to cater to observed scatter in experimental data of initiation time, time to initiation $\left(t_{I}\right)$ for a given $D$ is considered as a random variable following lognormal distribution. The mean and standard deviation of $\log \left(t_{I}\right)$ are given by:

$$
\left.\begin{array}{l}
\text { Mean value of } \log \left(t_{I}\right)=B_{0}+B_{1} \log (D) \\
\text { Standard deviation of } \log \left(t_{I}\right)=B_{2}+B_{3} \log (D)
\end{array}\right\} .
$$

$B_{0}, B_{1}, B_{2}$ and $B_{3}$ are constants whose values depend on type of material and loading conditions (i.e. constant load or changing load), and are evaluated by applying curve-fitting procedures to laboratory and field data. For AISI 304 austenitic stainless steel under constant load, $B_{0}=-3 \cdot 10, B_{1}=-4 \cdot 21, B_{2}=0 \cdot 3081$ and $B_{3}=0 \cdot 0$ (Harris et al 1992).

4.1b Crack size at initiation: In PRAISE, shape of surface crack initiated due to SCC is considered to be semi-elliptical, which is also consistent with shapes of stress corrosion cracks reported by Helie et al (1996) and Lu et al (2005). Surface length of initiated cracks, $(l=2 b)$, is assumed to be lognormally distributed with a median value of $1 / 8$ inch and standard deviation of $\log (b)$ as 0.85 (Harris et al 1992). Depth of initiated crack is taken to be $0.0254 \mathrm{~mm}(0.001 \mathrm{inch})$.

4.1c Multiple cracks: In materials subjected to SCC, many cracks would initiate successively and propagate simultaneously (Lu et al 2005), and hence multiple cracks can be present in a given weld. The expressions, given in PRAISE, for determining statistical properties of $t_{I}$, are mainly based on data from laboratory experiments on specimens about $50 \mathrm{~mm}$ long. Hence, these expressions are applicable to specimens of about $50 \mathrm{~mm}$ only. This is taken into account in PRAISE, by considering a given weld in the pipe to be composed of $50 \mathrm{~mm}$ segments adding up to length of weld. Initiation time for each segment is assumed to be independent and identically distributed.

4.1d Crack propagation due to SCC: It is assumed that initiated cracks grow at a constant velocity (initiation velocity, $v_{1}$ ) until conditions are appropriate for treating crack growth by fracture mechanics. The statistical properties of $v_{1}$ are determined using expressions given in PRAISE, which is obtained by correlating results of laboratory experiments with values of damage parameter $(D)$. In order to take into account considerable scatter in $v_{1}$ observed during experiments, $v_{1}$ is considered as a lognormally distributed random variable for a given $D$. While the standard deviation of $v_{1}$ is independent of $D$, mean of $\log \left(v_{1}\right)$ varies linearly with $\log (D)$, and is given by:

$$
\log \left(v_{1}\right)=F+G \log (D),
$$

where $F$ is normally distributed and $G$ is a constant. It can be noted that equation (13) is similar in form (power law) as that proposed by Helie et al (1996), based on experimental observations. For AISI 304 austenitic stainless steel, $F$ has a mean of 2.551 and standard deviation of 0.4269 , and $G=1.3447$ (Harris et al 1992). From parametric studies carried out, a value of $5 \%$ coefficient of variation of $\log \left(v_{1}\right)$ was found to be critical to life of piping component (Priya et al 2005), and hence this value is used in this study.

The procedure followed for transition from initiation to fracture mechanics crack growth rate in the present study is (Anoop et al 2008): 
(i) Pre-existing cracks always grow at fracture mechanics velocity.

(ii) Initiation velocity is always assigned to initiated cracks.

(iii) At any given time, if fracture mechanics velocity $\left(v_{2}\right)$ is greater than initiation velocity (i.e. $v_{2}>v_{1}$ ) and depth of crack is greater than $2.54 \mathrm{~mm}$, that particular crack grows at fracture mechanics velocity thereafter.

(iv) If the stress intensity factor for a crack is negative, the crack will not grow.

Fracture mechanics based crack growth velocity, $v_{2}$ (inches/year), is given by (Harris et al 1992):

$$
\log \left(v_{2}\right)=C_{14}+C_{15} D_{K},
$$

where $D_{K}$ is stress intensity factor $(K)$-related damage parameter given by

$$
D_{K}=C_{12} \log \left[f_{2} \text { (environment) }\right]+C_{13} K,
$$

where $C_{12}, C_{13}$ and $C_{15}$ are constants and $C_{14}$ is normally distributed. For AISI 304 austenitic stainless steel, $C_{12}=0.8192, C_{13}=0.03621$ and $C_{15}=1.7935$, mean value of $C_{14}=$ -3.1671 and standard deviation of $C_{14}=0.7260$ (Harris et al 1992).

From a probabilistic failure analysis of austenitic nuclear pipe against SCC, Priya et al (2005) inferred that expressions given in PRAISE for computation of stress intensity factors for modelling crack propagation need modification. In Priya et al (2005), this modification has been introduced by using well-accepted expressions given in ASM, and with modified PRAISE approach, stochastic propagation of stress corrosion cracks with time has been studied. It has been noted that trend of distribution of crack depths at initial stages is in satisfactory agreement with relevant experimental observations reported in literature (Priya et al 2005). Hence, modified PRAISE approach proposed by Priya et al (2005) is followed in the present study.

4.1e Coalescence of cracks: The multiple cracks that may be present can coalesce as they grow. Linking of two cracks takes place if spacing between them is less than the sum of their depths. After coalescence of two cracks, dimensions of modified crack are given by (Harris et al 1992)

$$
\left.\begin{array}{l}
\text { length, } l=l_{1}+S+l_{2} \\
\text { depth, } a=a_{1} \text { or } a_{2} \text {, whichever is greater }
\end{array}\right\} \text {, }
$$

where $l_{1}$ and $l_{2}$ are lengths of two cracks under consideration, $a_{1}$ and $a_{2}$ are crack depths and $S$ is spacing between them.

The initiation and propagation stages of stress corrosion cracks are modelled using modified PRAISE approach (Anoop et al 2008, Harris et al 1992, Priya et al 2005). The random variables, together with their distributions and their statistical properties, namely, mean and coefficient of variation (or standard deviation), are presented in table 2. To take into consideration the uncertainties arising due to the linguistic specifications of $T$ and $O_{2}$, these variables are represented by fuzzy sets with symmetric triangular membership functions. For engineering applications, to reduce computational complexity, fuzzy sets with triangular or trapezoidal form are most commonly used (Anoop et al 2006). Also, it has been shown by Dubois et al (1999) that symmetric triangular fuzzy sets are natural fuzzy counterparts to uniform probability distributions on bounded intervals, and hence, are the best probabilistic 
Table 2. Details of random variables considered. ${ }^{a}$

\begin{tabular}{|c|c|c|c|}
\hline Variable & Distribution & Parameters & Reference \\
\hline Applied stress $(\sigma)$ & Normal & $\begin{array}{l}\text { Mean }=105 \cdot 09 \\
\mathrm{COV}^{b}=0 \cdot 20\end{array}$ & Priya et al (2005) \\
\hline Yield strength $\left(\sigma_{y}\right)$ & Lognormal & $\begin{array}{l}\text { Mean }=151.53 \mathrm{MPa} \\
\mathrm{SD}^{c}=14.86 \mathrm{MPa}\end{array}$ & Rahman (1997) \\
\hline Ultimate strength $\left(\sigma_{u}\right)$ & Lognormal & $\begin{array}{l}\text { Mean }=450.63 \mathrm{KJm}^{-2} \\
\mathrm{SD}=25.53 \mathrm{KJm}^{-2}\end{array}$ & Rahman (1997) \\
\hline $\begin{array}{l}\text { Material fracture } \\
\text { toughness }^{d}\left(J_{I C}\right)\end{array}$ & Lognormal & $\begin{array}{l}\text { Mean }=1059 \cdot 56 \mathrm{KJm}^{-2} \\
\mathrm{SD}=450 \cdot 0 \mathrm{KJm}^{-2}\end{array}$ & Rahman (1997) \\
\hline
\end{tabular}

(Notes: ${ }^{a}$-In addition, degree of sensitization, time to crack initiation, initial crack length, crack initiation velocity and fracture mechanics based crack growth velocity are considered as random variables with parameters given in PRAISE manual (Harris et al 1992, Priya et al 2005).

$b_{-}$COV - Coefficient of variation

${ }^{c}$-SD - Standard deviation

${ }^{d}-K_{I C}$ is computed from $J_{I C}=K_{I C}^{2} / E^{\prime}$ where $E^{\prime}=E /\left(1-v^{2}\right)$ for plane strain, $v$ is Poisson's ratio, taken as $0 \cdot 3$ )

approximation. The specified values for the fuzzy variables, given in table 1, are taken as the corresponding prototypical elements (one and only element with grade of membership equal to 1.0). Since authors could not get information on variation of $T$, support of fuzzy set of operating temperature is determined assuming a variation of $\pm 10 \%$ about the specified value. A higher variation of $\pm 20 \%$ about the specified value is chosen for determining support of fuzzy set of $\mathrm{O}_{2}$ since it is found to vary over a significant range (Lin 1996). The membership functions of different fuzzy variables are shown in figure 2 .

The rate of reduction in pipe wall thickness due to SCC increases with increase in operating temperature $(T)$ and oxygen concentration $\left(\mathrm{O}_{2}\right)$. Therefore, $P_{F}$ can be considered as a monotonic function of $T$ and $O_{2}$, and hence the proposed procedure can be used for determining the bounds on $P_{F}$. The R6 procedure with option 1 curve (Ainsworth 1996) is used for safety assessment. Reference stress values $\left(\sigma_{\text {ref }}\right)$, required in R6 procedure, are determined using the expressions given in API (2000). Flow stress $\left(\sigma_{f}\right)$ is computed as the average value of yield strength $\left(\sigma_{y}\right)$ and ultimate strength $\left(\sigma_{u}\right)$. Following assumptions are made in the analysis:

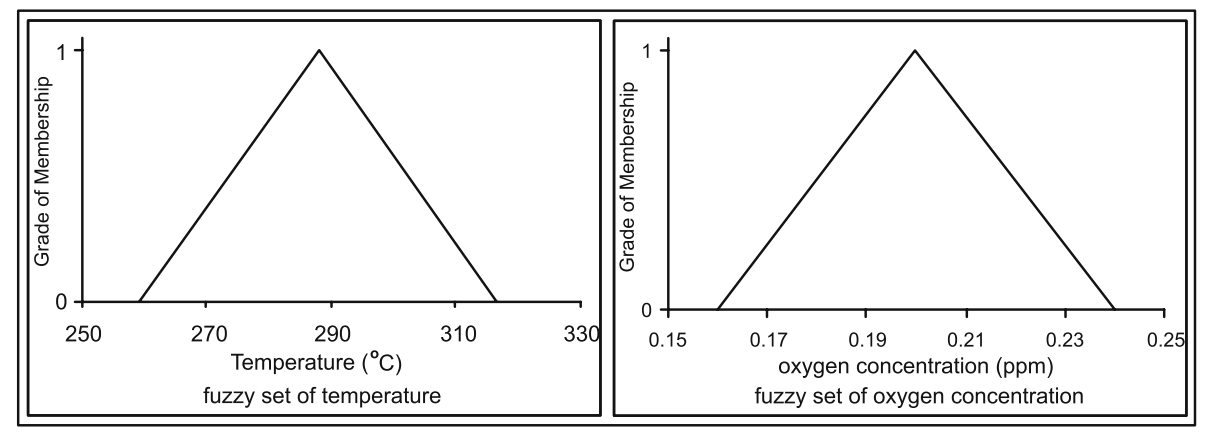

Figure 2. Fuzzy sets of operating temperature and oxygen concentration. 
- Variation in material properties (namely, material fracture toughness, yield strength, ultimate strength and modulus of elasticity) with change in temperature, for the range of operating temperature considered (figure 2), is neglected.

- From the results of statistical analysis reported by Rahman (1997), it is noted that the degree of correlation between yield strength and ultimate strength of AISI 304 stainless steel is small (i.e. correlation coefficient $=0 \cdot 31$ ). Hence, yield strength and ultimate strength are assumed to be statistically independent.

- Modulus of elasticity of AISI 304 stainless steel is considered to be deterministic.

In the present study, Monte Carlo simulation method is used for determining the failure probability. Ten thousand simulation cycles are used for each combination of CDF bounds of the fuzzy variables (total number of combinations $=2^{m}=4$, since there are two fuzzy variables in this case), and inverse transformation technique is used for the generation of values of random variables corresponding to the CDFs considered.

For the purpose of comparison, probabilistic analyses are also carried out by: (i) without considering fuzzy uncertainties, taking values of $T$ and $\mathrm{O}_{2}$ as the specified values (hereafter this case is referred to as Probabilistic Analysis I), and, (ii) by considering $T$ and $\mathrm{O}_{2}$ as random variables (instead of fuzzy variables) following symmetric triangular probability distributions with same range as that of corresponding fuzzy set (hereafter this case is referred to as Probabilistic Analysis II).

\section{Results and discussion}

The aspect ratio of cracks (defined as the ratio of crack depth to half crack length) that corresponds to failure at different times, for the four combinations of the CDFs considered, are shown in figure 3 . From the dimensions of the cracks corresponding to failure, it is noted that the coalesced cracks are the ones which contribute to failure, i.e. the initiated cracks coalesce as they grow, and further growth of these coalesced cracks lead to failure.

The variation in $P_{F}$ with time corresponding to four combinations of the CDFs are shown in figure 4. From this figure, it is noted that the combination corresponding to upper-bound CDFs of $T$ and $\mathrm{O}_{2}$ gives lower values of $P_{F}$ while that corresponding to lower-bound CDFs gives higher values of $P_{F}$. This is expected because use of lower-bound CDFs result in generation of variables with higher values, and since rate of reduction in pipe wall thickness due to SCC increases with increase in $T$ and $\mathrm{O}_{2}$, combination of lower-bound CDFs of $T$ and $\mathrm{O}_{2}$ gives higher values of $P_{F}$.

The effect of coalescence of cracks on failure probability is shown in figure 5. It is noted from this figure that, as expected, an increase (or decrease) in the number of coalescing cracks result in an increase (or decrease) in the rate of failure probability in the near future (within 3 to 4 years). Thus, the plateau observed in the time versus failure probability curves (as seen in figures 4 and 5) can be attributed to the decrease in the number of coalescing cracks.

The bounds on $P_{F}$ obtained from the proposed procedure along with the $P_{F}$ values obtained using Probabilistic Analyses I and II are shown in figure 6. It is noted that values of $P_{F}$ obtained using Probabilistic Analyses I and II are more or less in agreement with each other, indicating that the random variations in $T$ and $O_{2}$ do not affect failure probability significantly. It is also noted that, as expected, bounds on $P_{F}$ obtained using the proposed procedure encloses the $P_{F}$ values obtained from Probabilistic Analyses I and II. While it is possible to determine the bounds on $P_{F}$ by carrying out a probabilistic analysis, the bounds obtained using the proposed procedure are more rational since appropriate representations of uncertainty 


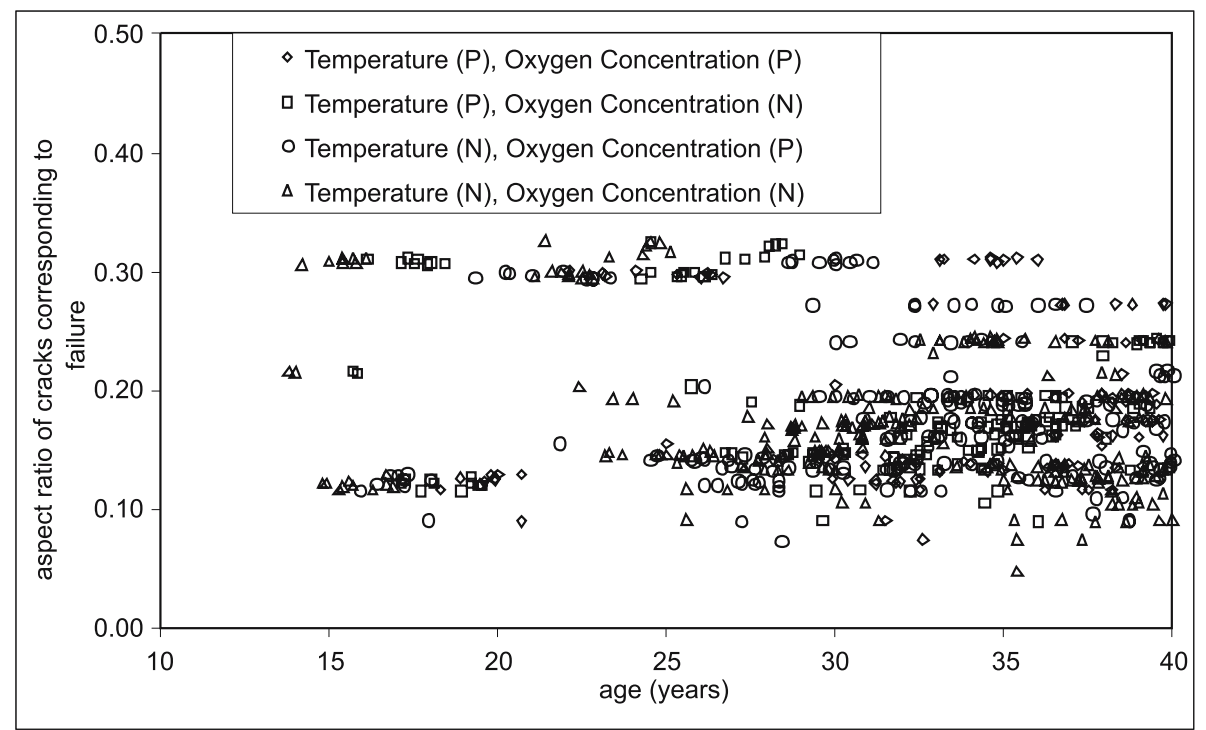

Figure 3. Aspect ratio of cracks corresponding to failure at different times for the four combinations of lower- and upper-bound CDFs considered ((P) - upper-bound CDF corresponding to possibility measure; $(\mathrm{N})$ - lower-bound $\mathrm{CDF}$ corresponding to necessity measure).

are used for the different variables, and hence are consistent with the available information. The upper-bound for $P_{F}$ shown in figure 6 can be used in decision-making. For instance, at the design stage, the upper-bound curve can be used along with the specified target $P_{F}$

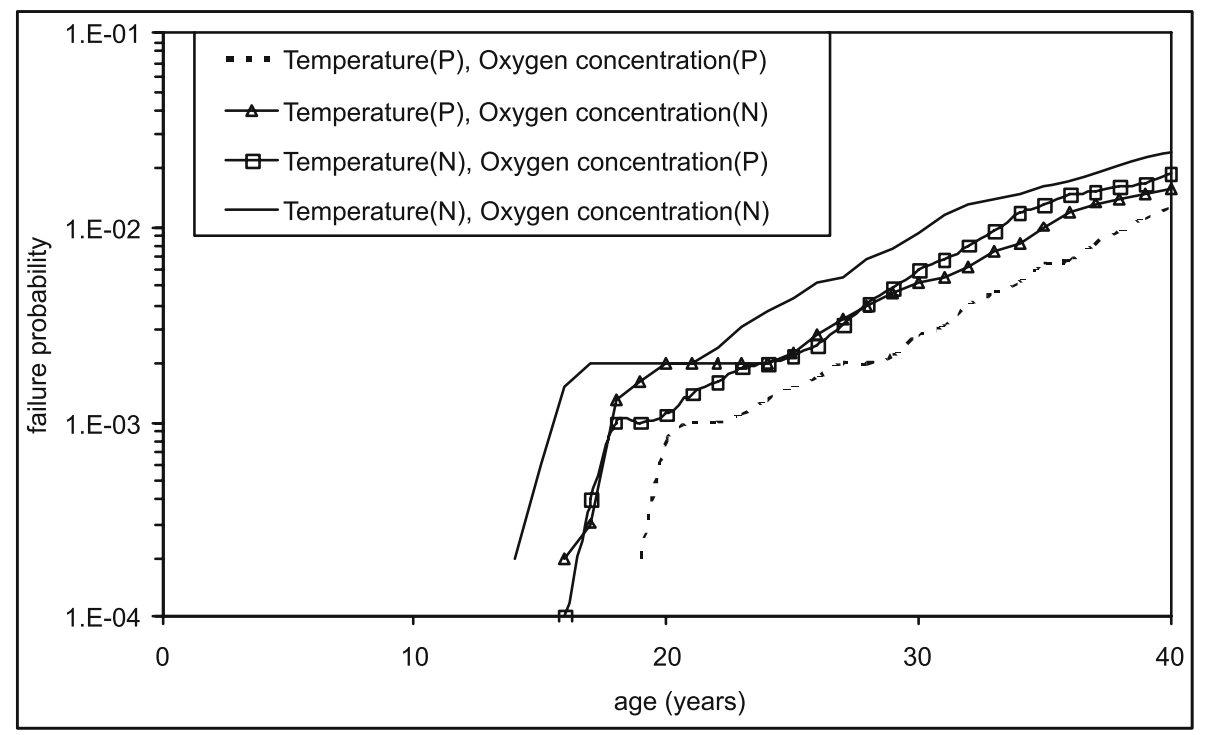

Figure 4. Variation in $P_{F}$ with time for the four combinations lower- and upper-bound CDFs considered for the piping component subjected to stress corrosion cracking $((\mathrm{P})$ - upper-bound CDF corresponding to possibility measure; $(\mathrm{N})$ - lower-bound $\mathrm{CDF}$ corresponding to necessity measure). 


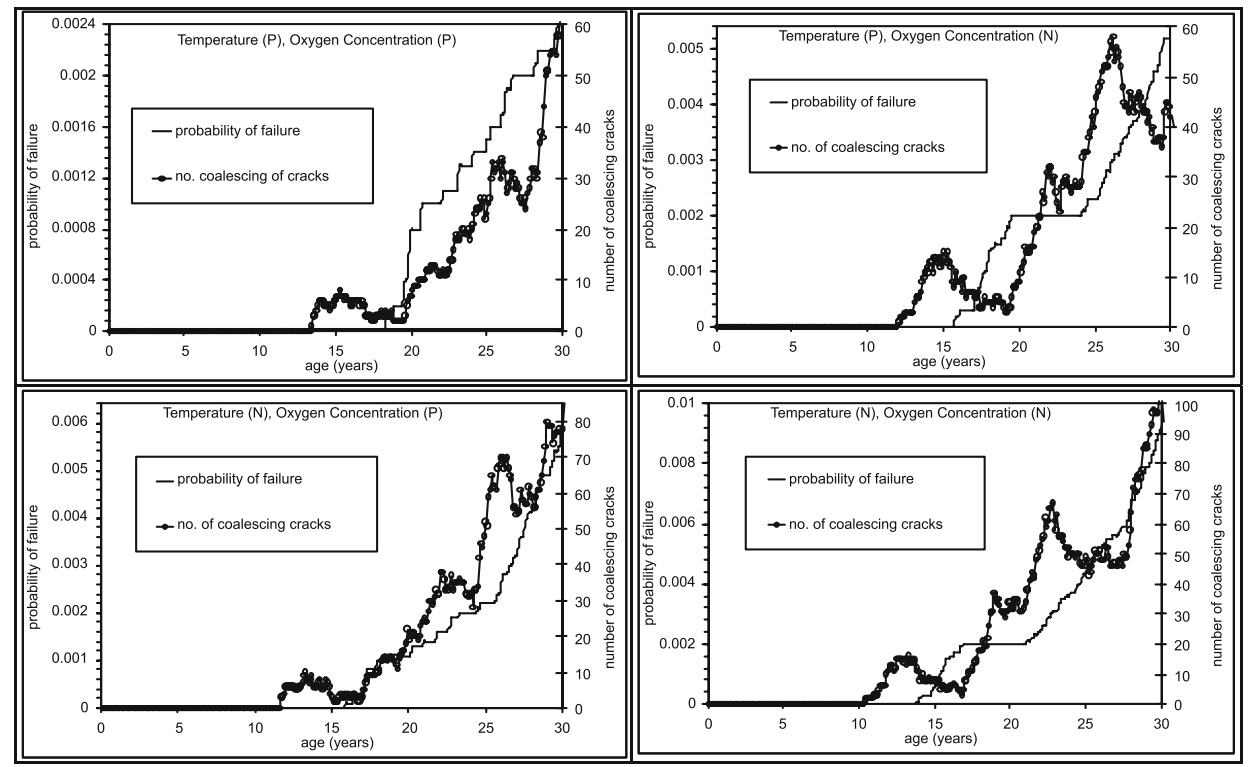

Figure 5. Variation in $P_{F}$ and number of coalescing cracks with time for the four combinations of lower- and upper-bound CDFs considered ((P) - upper-bound CDF corresponding to possibility measure; $(\mathrm{N})$ - lower-bound CDF corresponding to necessity measure).

values for design decision-making. For existing power plants, the $P_{F}$ value can be combined with conditional core damage probability associated with SCC failure of piping component to determine contribution towards plant core damage probability. Carrying out this type of

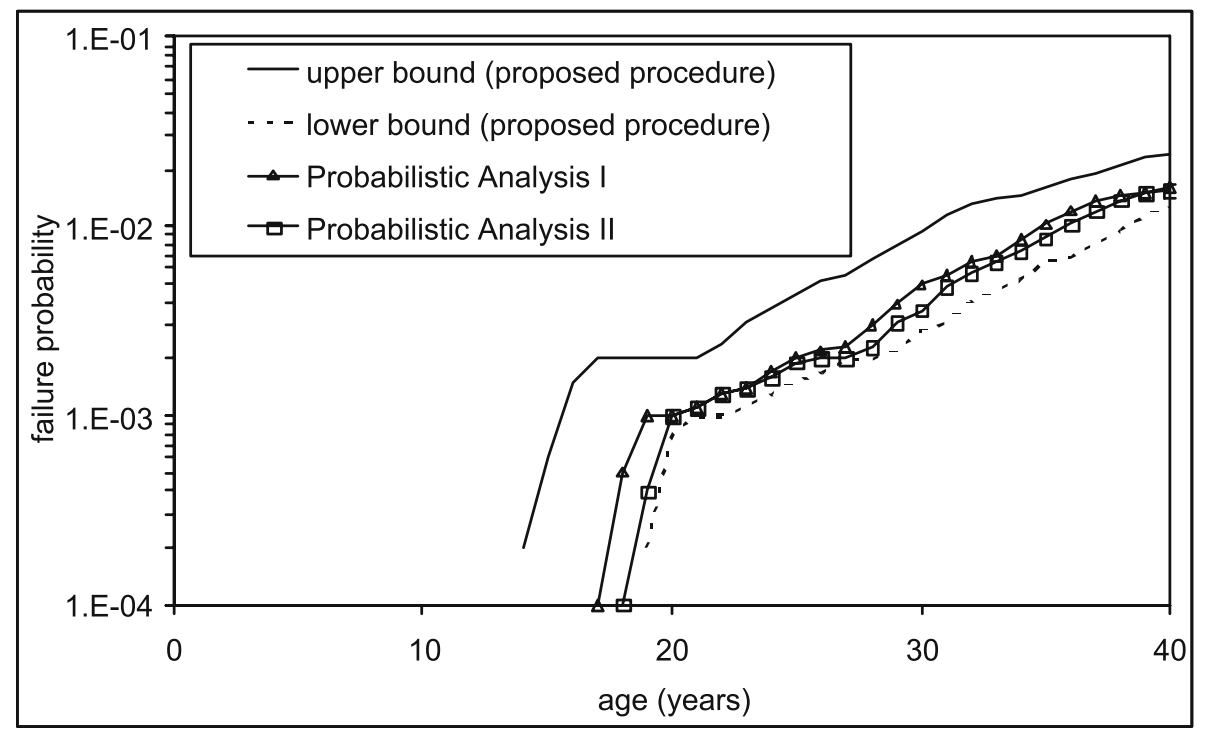

Figure 6. Comparison of bounds for $P_{F}$ obtained using proposed procedure with the $P_{F}$ values obtained using probabilistic approaches for the piping component subjected to stress corrosion cracking. 
analysis for different types of piping components susceptible to SCC will help in ranking them for risk-informed, in-service inspection and maintenance activities.

\section{Conclusions}

A procedure for safety assessment in the presence of probabilistic and fuzzy uncertainties is presented. The procedure can be used for determining the bounds on failure probability for cases where the failure probability is a monotonic function of the variables considered as fuzzy. The usefulness of the proposed procedure is illustrated through an example problem of safety assessment of a nuclear power plant piping component against stress corrosion cracking, considering stochastic evolution of stress corrosion cracks with time. It is noted that the bounds on failure probability obtained using the proposed procedure encloses the values of failure probability obtained from probabilistic analysis. From the results obtained, it is noted that the approach presented shows promise for safety assessment of engineering facilities, such as nuclear power plants.

This paper is being published with the kind permission of the Director, Structural Engineering Research Centre, Chennai.

\section{References}

Ainsworth R A 1996 Failure assessment diagrams for use in R6 assessments for austenitic components. Int. J. Pres. Vessels Piping 65(3): 303-309

Anoop M B, Balaji Rao K, Gopalakrishnan S 2006 Conversion of probabilistic information into fuzzy sets for engineering decision analysis. Comp. and Struct. 84(3-4): 141-155

Anoop M B, Balaji Rao K, Lakshmanan N 2008 Safety assessment of austenitic steel nuclear power plant pipelines against stress corrosion cracking in the presence of hybrid uncertainties. Int. J. Pres. Vessels Piping. 85(4): 238-247

API 2000 API 579: Recommended practice for fitness-for-service. (Washington DC: American Petroleum Institute)

Chen S, Nikolaidis E, Cudney H H, Rosca R, Haftka R T 1999 Comparison of probabilistic and fuzzy set methods for designing under uncertainty. In: 40th AIAA/ASME/ASCE/AHS/ASC Structures, Structural Dynamics and Materials Conference. St. Louis

Dubois D, Prade H 1980 Fuzzy Sets and Systems: Theory and Applications. (San Diego: Academic Press Inc.)

Dubois D, Prade H, Sandri S 1993 On possibility/probability transformations. In: R Lowen, M Roubens (Eds.) Fuzzy Logic. (Dordrecht: Kluwer Academic Publishers)

Dubois D, Prade H, Yager R 1999 Merging fuzzy information. In: J C Bezdek, D Dubois, H Prade (Eds.) Fuzzy sets in Approximate Reasoning and Information Systems. (Dordrecht: Kluwer Academic Publishers)

Ferrari P, Savoia M 1998 Fuzzy number theory to obtain conservative results with respect to probability. Comput. Meth. Appl. Mech. Eng. 160: 205-222

Haldar A, Reddy R K 1992 A random-fuzzy analysis of existing structures. Fuzzy Sets Syst. 48(2): 201-210

Harris D O, Dedhia D D, Lu S C 1992 Theoretical and User's Manual for pc-PRAISE. NUREG/CR5864 
Helie M, Peyrat C, Raquet G, Santarini G, Sornay Ph 1996 Phenomenological modelling of stress corrosion cracking. Intercorr/96 First Global Internet Corrosion Conference. http://www.corrosionsource.com/events/intercorr/techsess/papers/session3/abstracts/helie.html

Helton J C, Oberkampf W L 2004 Alternative representations of epistemic uncertainty. Reliab. Engng. Syst. Safety 85(1-3): 1-10

Lin C C 1996 Radiochemistry in nuclear power reactors. (Washington DC: National Academy Press)

Lu B T, Chen Z T, Luo J L, Patchett B M, Xu Z H 2005 Pitting and stress corrosion cracking behaviour in welded austenitic stainless steel. Electrochimica Acta 50(6): 1391-1403

Nikolaidis E, Haftka R T 2001 Theories of uncertainty for risk assessment when data is scarce. Int. J. Adv. Manufact. Syst. 4(1): 49-56

Priya C, Balaji Rao K, Lakshmanan N, Gopika V, Kushwaha H S, Saraf R K 2005 Probabilistic failure analysis of austenitic nuclear pipelines against stress corrosion cracking. Proc. Inst. Mech. Eng. Part C: J. Mech. Eng. Sci. 219(7): 607-626

Rahman S 1997 Probabilistic failure analysis of cracked pipes with circumferential flaws. Int. J. Pres. Vessels Piping 70(3): 223-236

Ross T J 1995 Fuzzy logic with engineering applications. (New York: McGraw-Hill Inc)

Savoia M 2002 Structural reliability analysis through fuzzy number approach, with application to stability. Comp. and Struct. 80(12): 1087-1102 